\title{
Proton kinetics through the cuticle layer in maize
}

\author{
Monika Olszewska $^{1} \cdot$ Adam Konefal $^{2} \cdot$ Mariusz Pietruszka ${ }^{1}$
}

Received: 24 November 2016/Revised: 1 July 2017/Accepted: 15 October 2017/Published online: 23 October 2017

(C) The Author(s) 2017. This article is an open access publication

\begin{abstract}
A Monte Carlo simulation was used to determine the dependence between the thickness of the cuticle layer of coleoptiles and the spectra of the $\mathrm{H}^{+}$ions (i.e., protons) passing through this layer, which is treated as a potential barrier. The apparently simplistic model of a walled cylinder filled with $\mathrm{H}^{+}$ions propagating through the cuticle layer was solved in silico. We showed that the thickness of the cuticle layer clearly influences the intensity of the efflux of protons, which changes the $\mathrm{pH}$ of the surrounding solution. Then, diffusion and cross-correlation data for maize (Zea mays L.) coleoptile growth and $\mathrm{H}^{+}$ion extrusion were probed in an experiment and compared with the Monte Carlo computation results. Ex vivo experiments for the control (APW), auxin (IAA) and fusicoccin (FC) were conducted. The transition from time-delayed $\mathrm{pH}-$ (abrasion time) cross-correlation to proton efflux that was not retarded was obtained, thus confirming the canvas that constitutes the acid growth hypothesis and the rationale that was accepted for the derivation of the 'equation of state' for plants.
\end{abstract}

Communicated by U. Feller.

Electronic supplementary material The online version of this article (doi:10.1007/s11738-017-2559-6) contains supplementary material, which is available to authorized users.

Mariusz Pietruszka

mariusz.pietruszka@us.edu.pl

1 Department of Plant Physiology, Faculty of Biology and Environment Protection, University of Silesia, Jagiellońska 28, 40032 Katowice, Poland

2 Department of Nuclear Physics and Its Applications, Institute of Physics, University of Silesia, Katowice, Poland
Keywords APW · Auxin - Acid growth · Coleoptile · Fusicoccin $\cdot \mathrm{H}^{+}$ions $\cdot$ Monte Carlo simulation - Proton efflux $\cdot \mathrm{pH} \cdot$ Potential barrier $\cdot$ Proton scattering . Zea mays L.

\section{Introduction}

\section{Acid growth and plant development}

The wall acidification model, which was proposed in 1971 by Hager-the spiritual father of the acid-growth concept-(Hager 2003) is based on experiments with shoots of grass seedlings (coleoptiles, which are leaf-like axial organs). The hypothesis has been evaluated by many scientists since then (e.g., Kutschera 1994, 2003; Lüthen et al. 1990) and is still a matter of debate (Cosgrove 2001, 2005; Lüthen 2015). Plant growth also depends on biomechanics, wall structure and cell wall modification proteins (Braidwood et al. 2014; Bidhendi and Geitmann 2015). Recently, a cross-correlation analysis of acid growth data (Pietruszka and Haduch-Sendecka 2016a) led us to initial conclusions concerning the potential role of the cuticle layer in growth medium acidification. These preliminary results turned out to be conjugated with the phenomenological equations put forward by Pietruszka (2017). Indeed, the 'equation of state' and equation of evolution for the extending primary cell wall are in agreement with the results of the calculations for the cross-correlations (Pietruszka and Haduch 2016a, b). In fact, these results formed a canvas for the 'state' (describing wall properties) and evolution equations. Nonetheless, it is clearly visible (ibid.) that $\mathrm{pH} /$ growth cross-correlations differ greatly depending on sample preparation. This means that the outcomes for abraded and non-abraded samples varied, among others, in the time 
delay of the $\mathrm{H}^{+}$ions efflux. Therefore, the main purpose of this work was to establish the strict relations of $\mathrm{pH}$-induced growth, parameterised by the cuticle layer potential barrier (by its thickness). An analysis of our recent data (ibid.) suggested a transition from a retarded to a quasi-immediate response of the system. The latter was confirmed in both the theoretical and experimental methods presented in this work.

\section{Materials and methods}

\section{Model calculations}

In this work, GEANT4 Monte Carlo software version 10.2.p01 with the GEANT4-DNA physical process libraries was used to determine the dependence between the thickness of the cuticle layer and the spectra of $\mathrm{H}^{+}$ ions passing through this layer. GEANT4 consists of a broad collection of $\mathrm{C}++$ class libraries that users can use to build their own simulation program, which gives users unrestricted control over the simulation. The early versions of the software were dedicated to applications in high-energy physics. Recently, the range of GEANT4 applications was widened to the other branches of science. In particular, the GEANT4 Monte Carlo simulation toolkit has been extended with processes for modelling the interactions that are induced by ionising low-energy radiation at the molecular (DNA) level. These interaction models were implemented in the simulation program to calculate the above-mentioned dependence for $\mathrm{H}^{+}$ions. These models make it possible to simulate protons such as $\mathrm{H}^{+}$ions with a relatively low energy of $100 \mathrm{eV}$ (see Supplementary Information for details). The simulations were carried out for three thicknesses of the cuticle layer $-1,0.5$ and $0.1 \mu \mathrm{m}$. The scheme of the simulated geometry is presented in Fig. 1. Two situations were considered. In the first approach, calculations were performed for an isotropic proton source. The position of the source was a random variable in the volume inside the cuticle layer. Such an approach makes it possible to obtain an isotropic distribution of the directions of the $\mathrm{H}^{+}$ ion hits in the barrier. In the second approach, the direction of the $\mathrm{H}^{+}$ion propagation was changed so that it was perpendicular to the surface of the cuticle layer. The simulations were carried out using the Ubuntu 12.04 operating system in the computer cluster in the Department of Nuclear Physics and Its Applications in the Institute of Physics of the University of Silesia in Katowice (Poland). The calculations were run at $10^{8} \mathrm{H}^{+}$ ions hitting the barrier per hour on a $3-\mathrm{GHz}$ Pentium computer cluster.

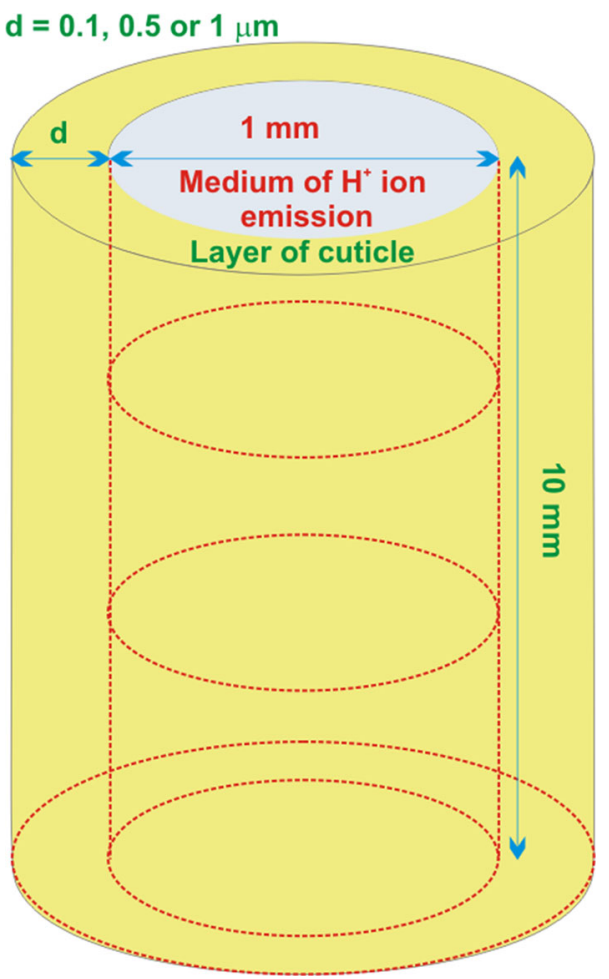

Fig. 1 Scheme of the geometry of the simulated system

\section{Experimental procedure}

\section{Plant material}

Experiments were done using 3-day-old seedlings of Zea mays $\mathrm{L} \mathrm{cv}$. Cosmo. Maize seeds were soaked in tap water for $2 \mathrm{~h}$ and then sown in moist lignin. The seedlings were grown in an incubator in darkness at $27 \pm 0.5^{\circ} \mathrm{C}$. Ten-mm segments were cut from the plants $5 \mathrm{~mm}$ below the tip and first leaf was removed. Depending on the experimental variant, the cuticle of the coleoptile segments was abraded or remained untouched (control). The average weight of the coleoptile segments before and after the experiment is presented in SI Table 1 (average increase by about $10 \%$ after $8 \mathrm{~h})$.

\section{Abrasion of the cuticle}

The cuticle abrasion method was prepared partially based on the article by Lüthen et al. (1990). About $0.9 \mathrm{~g}$ of tissue (i.e., 40-45 segments) was placed in a $150 \mathrm{ml}$ beaker with a 30-ml incubation solution, artificial pond water, APW $(0.1 \mathrm{mM} \mathrm{NaCl}, 0.1 \mathrm{mM} \mathrm{CaCl} 2,1 \mathrm{mM} \mathrm{KCl}$ with $\mathrm{pH} 6.5$ adjusted with $\mathrm{HCl}$ or $\mathrm{NaOH}$ ) containing $0.3 \mathrm{~g}$ of $\mathrm{SiC}$ powder (600 mesh). The beaker containing the coleoptiles and abrasive powder was mixed at a rate of 250 RPM in a magnetic stirrer. Scrubbing time depended on the 
experimental variant (SI Figure 1). The segments of coleoptiles were washed with distilled water after abrasion and inserted into a measuring device. The variants differed in the duration of the abrasion time with the $\mathrm{SiC}$ powder on a magnetic stirrer (SI Figure 2A): I-0 min (control), II$2 \mathrm{~min}, \mathrm{III}-3 \mathrm{~min}, \mathrm{IV}-5 \mathrm{~min}, \mathrm{~V}-6 \mathrm{~min}, \mathrm{VI}-8 \mathrm{~min}$ and VII-10 min (largest abrasion).

\section{Measurement of growth and acidification}

Experiments were performed for three cases: for APW, indole-3-acetic acid, the biologically active form of auxin (IAA) at a concentration of $10^{-4} \mathrm{M}$ and fusicoccin (FC) at a concentration of $10^{-6} \mathrm{M}$. In the latter two cases, the growth stimuli IAA and FC were introduced into the incubation medium after $2 \mathrm{~h}$. Forty 10-mm-long coleoptile segments were prepared for each variant and then placed in two identical sets (20) of the elongation-measuring apparatus (Fig. 2 and SI Figure 2B) in an aerated incubation medium. The volume of the incubation medium in the apparatus was $6 \mathrm{ml}$ per glass tube $(0.3 \mathrm{ml}$ of the incubation medium per segment). Measurements of the growth and $\mathrm{pH}$ were performed for $8 \mathrm{~h}$. The $\mathrm{pH}$ measurement was carried out using two CPI-501 pH-meters (separately for each tube). The temperature during the experiment was $25 \pm 0.5^{\circ} \mathrm{C}$ and was maintained using a water bath at a similar level. All manipulations were performed under dim green light. The shadow-graph method was used with an $\times 18$ magnification. The optical system that was used for the growth measurements permitted the longitudinal extension of a stack of 20 segments to be recorded simultaneously. Changes in elongation $[\mu \mathrm{m}]$ were expressed as the differences between the length of the coleoptile segments at a given time during the experiment and their initial length. Only one of the two tests in separate tubes was selected for analysis. Growth and $\mathrm{pH}$ were recorded every $15 \mathrm{~min}$. Segment elongation at a magnification of $\times 18$ was recorded on a millimetre scale with an accuracy of $\pm 0.5 \mathrm{~mm}$. The method for measuring the elongation growth of the segments of maize was used, e.g., by Pietruszka and Lewicka (2007).

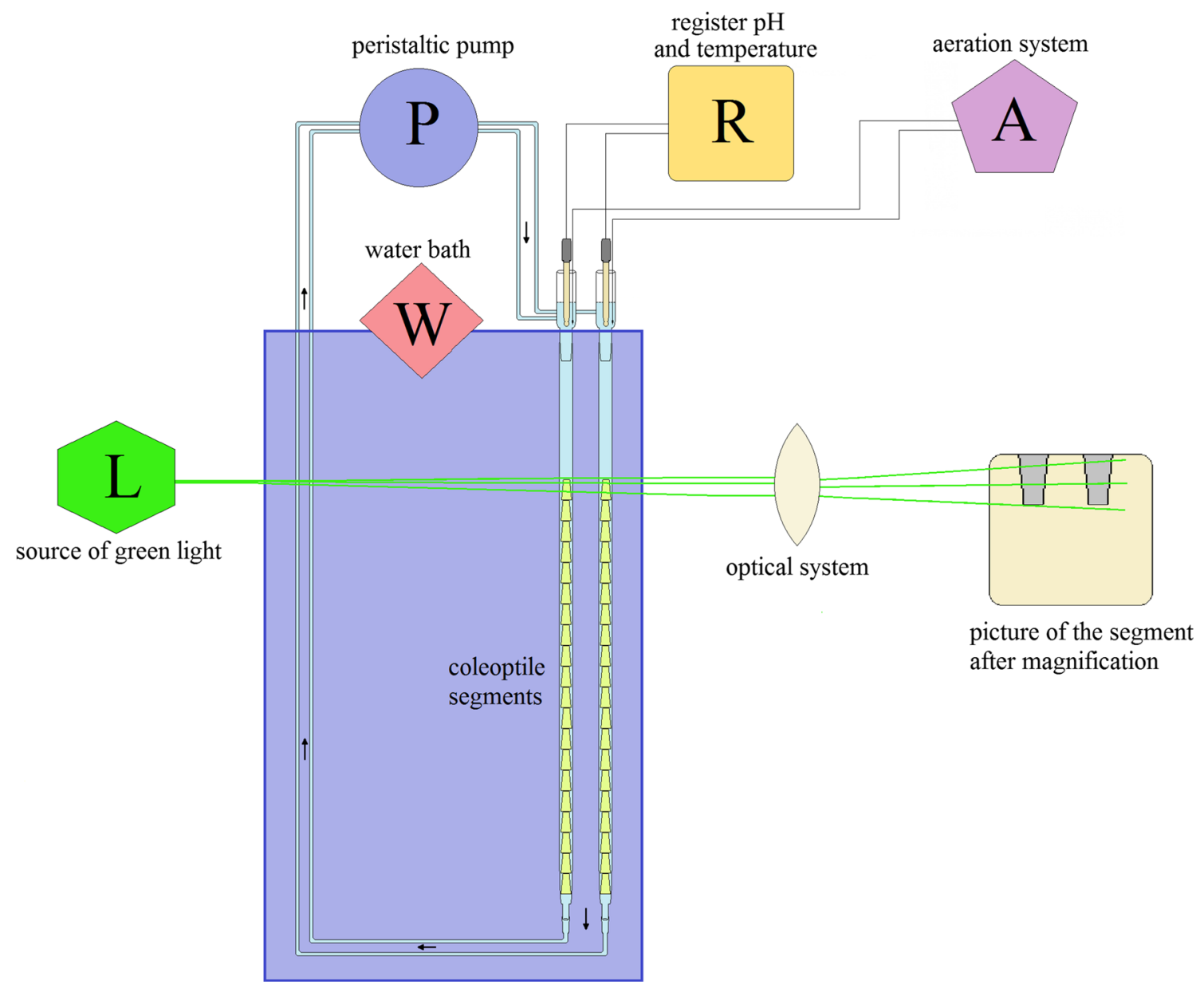

Fig. 2 The experimental setup 
Scanning electron microscopy (SEM)—sample preparation

Three methods of sample preparation for SEM were tested: (1) Amylacetate, (2) Glutaraldehyde, (3) Methanol. The method of glutaraldehyde was chosen for best results. Segments were fixed for $5 \mathrm{~min}$ in $1.5 \%$ (v/v) glutaraldehyde buffer (phosphate-buffered glutaraldehyde for $30 \mathrm{~s}$ ). Then aqueous $\mathrm{OsO}_{4}$ was added to a final concentration of $1 \%(\mathrm{w} / \mathrm{v})$ and fixed for the additional $5 \mathrm{~min}$. The segments were dehydrated in a graded ethanol series: $30,50,70,80$, $90,95 \%$ and $2 \times 100 \%$ and subjected to critical point drying using liquid $\mathrm{CO}_{2}$ (Pelco CPD2). The segments were coated with gold $(19.2 \mathrm{~nm})$ in Pelco SC-6 and observed at $5 \mathrm{keV}$ using Hitachi UHR FE-SEM SU 8010 scanning electron microscope.

\section{Staining with Evans blue and neutral red}

To show the impact of abrasion $0.5 \%$ Evans blue water solution, which stains the dead cells, was applied. The staining was needed as the stirrer might mechanically impact the segments or even damage. This kind of staining, in general, also allows to critically optimize all parameters (abrasion time, SiC particle size, $\mathrm{SiC}$ concentration, vessel geometry, stirrer speed, etc.).

To demonstrate the efficiency of the abrasion method, neutral red $1 \mathrm{~g} / \mathrm{l}$ with acetic acid $2 \mathrm{ml} / \mathrm{l}$ in water was applied. It shows how many cells are actually opened to the external solution and how homogeneous the action of the $\mathrm{SiC}$ on the surface is.

\section{Cross-correlation analysis}

In signal processing, cross-correlation is a measure of the similarity of two waveforms as a function of a time lag that is applied to one of them. This is also known as a sliding dot product or sliding inner product. For continuous functions $f$ and $g$, the cross-correlation is defined in Eq. (1) by the integral

$(f * g)(\tau) \equiv \int_{-\infty}^{\infty} f^{*}(t) g(t+\tau) \mathrm{d} t$,

where $f^{*}$ denotes the complex conjugate of $f$ and $\tau$ is the time lag (here $f^{*}$ and $f$ are equal). Note that the crosscorrelation is maximum at a lag that is equal to the time delay.

\section{Relative elongation growth formula}

For growth analysis, we used the experimental data for the double-exponent growth functional (Pietruszka 2012),
Eq. (2), which was elaborated for practical use in Zajdel et al. (2016) for the relative (volumetric) growth rate

$\frac{V_{T}(t)-V_{0}}{V_{0}}=A t+B+C(T) e^{-e^{-D(T)(t-t e)}}$,

where $A, B, C$ and $D$ are positively defined coefficients that are constant in time. The coefficients $C=C(T)$ and $D=D(T)$ are, in principle, temperature ( $T$ ) dependent (in our case we put $T=$ const.); $C$ denotes the amplitude, while $D$-the diffusion rate. A particular time $t=t_{e}$ stands for the inflection point (corresponding to the maximum in the growth rate) of the sigmoid-like growth curve and $V_{0}=V(t=0)$ is the initial volume. In the present analysis, the effective diffusion rate, which involves net ions transport to the wall, was our main interest since it can be directly inferred from the fits. Its value, which depends on dimensionality such as $[D]=1 / \mathrm{s}$, can be compared between different experimental conditions. Note that the coefficient $D$ (diffusion rate) should not be confused with the diffusion constant $D$ that enters the Fick's 1st law.

\section{Results}

\section{Monte Carlo simulation}

The spectra of the $\mathrm{H}^{+}$ions passing through the cuticle barrier at a density of $0.1 \mathrm{~g} / \mathrm{cm}^{3}$ were calculated (Figs. 3, 4). The events were registered in $10 \mathrm{eV}$ energy bins, which made it possible to obtain a satisfactory resolution. The total number of $\mathrm{H}^{+}$ions shot at the barrier to derive one spectrum with statistical fluctuations less than $0.5 \%$ was $10^{9}$ (one standard deviation level). In each spectrum, two components were visible. The low-energy component predominated for the $0.1 \mu \mathrm{m}$ thick cuticle layer, whereas the higherenergy one was dominant for the two other thicknesses of the barrier that were considered (Fig. 4).

The simulations that were performed for the isotropic source and the mono-directional one indicated that the direction of the $\mathrm{H}^{+}$ion before hitting the barrier does not influence the shape of the spectrum (Fig. 3).

A strong fall off of the number of $\mathrm{H}^{+}$ions passing through the barrier was visible in the wax layer thicknesses from $0.1 \mu \mathrm{m}$ to $0.3 \mu \mathrm{m}$ (Fig. 5). The intensity of the $\mathrm{H}^{+}$ ions was almost constant for the thicker barriers. A cuticle layer of $1.2 \mu \mathrm{m}$, comparable to the thickness of cell wall, was needed to stop all of the $\mathrm{H}^{+}$ions.

$\mathrm{H}^{+}$ions primarily lose their energy through a multiple scattering process. In this electromagnetic interaction, the $\mathrm{H}^{+}$ions interact with the nuclei of the matter as well as 
Fig. 3 Comparison of the calculated spectra of $\mathrm{H}^{+}$ions passing through the cuticle barrier for the isotropic source and the mono-directional ion source. The calculations were performed for $\mathrm{H}^{+}$ions with the initial energy of $100 \mathrm{eV}$ for the $0.1-\mu \mathrm{m}$-thick barrier
Fig. 4 The $\mathrm{H}^{+}$ion spectra obtained for various thicknesses of the cuticle barrier
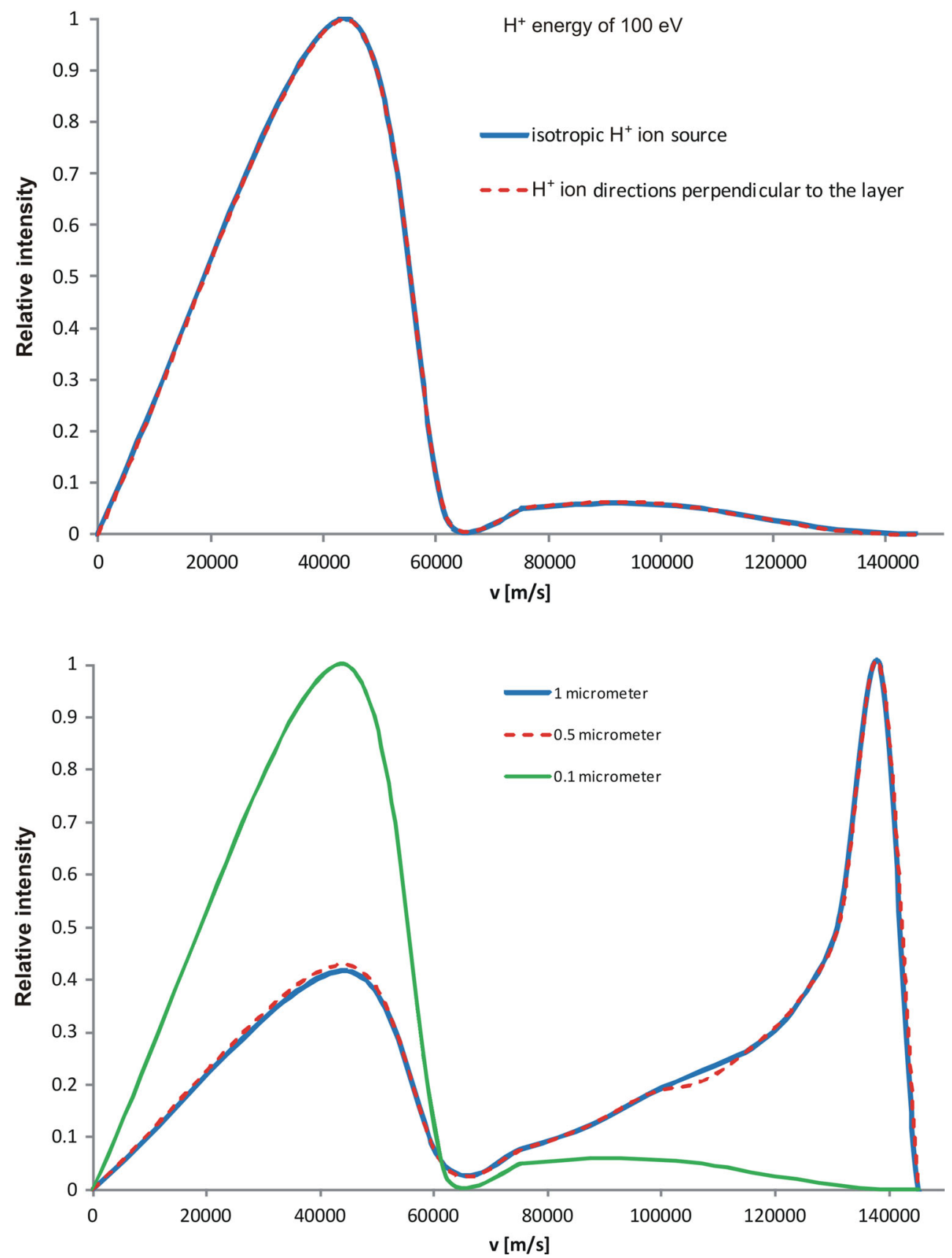

with the electrons of the atoms. Depending on the number of single acts of the scattering processes, the protons can lose different amounts of energy inside the statistical fluctuations. The low-energy component of the obtained spectra corresponded to a larger number of scattering acts. The spatial structure of the simulated system in terms of the distance covered by a $\mathrm{H}^{+}$ion in the cuticle barrier is presented schematically in Fig. 6.

\section{Cuticle abrasion experiment}

Simultaneous measurements of elongation growth and $\mathrm{pH}$ (Fig. 2) in control conditions (APW) and for IAA and FC on Zea mays L. (maize) segments were performed for all seven variants (SI Figure 1) at $25 \pm 0.5^{\circ} \mathrm{C}$.

The time cross-sections of $\mathrm{pH}$ measured as a function of the cuticle layer thickness for APW (IAA, FC) are presented in Figs. 7, 8 and 9, respectively. After the first $2 \mathrm{~h}$ of the experiment, a linear decrease of $\mathrm{pH}$ along with the abrasion time was noticeable in all cases (variants). Interestingly, the linear slope and intercept values changed 


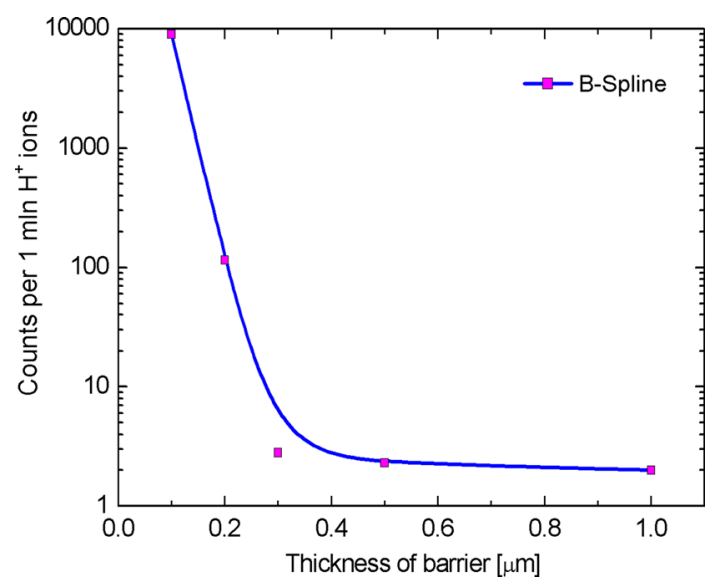

Fig. 5 The dependence between the number of $\mathrm{H}^{+}$ions passing through the cuticle barrier (log vertical scale) and the thickness of the cuticle layer. The number of protons that survived (creating $\mathrm{H}^{+}$efflux into the external medium) is approximately indicated by the solid (blue) line, e.g., for $0.2 \mu \mathrm{m}$ cuticle layer $0.1 \%$ of incident ions survives. Solid (magenta) dots-Monte Carlo simulation; interpolated by B-spline (Microcal Origin)

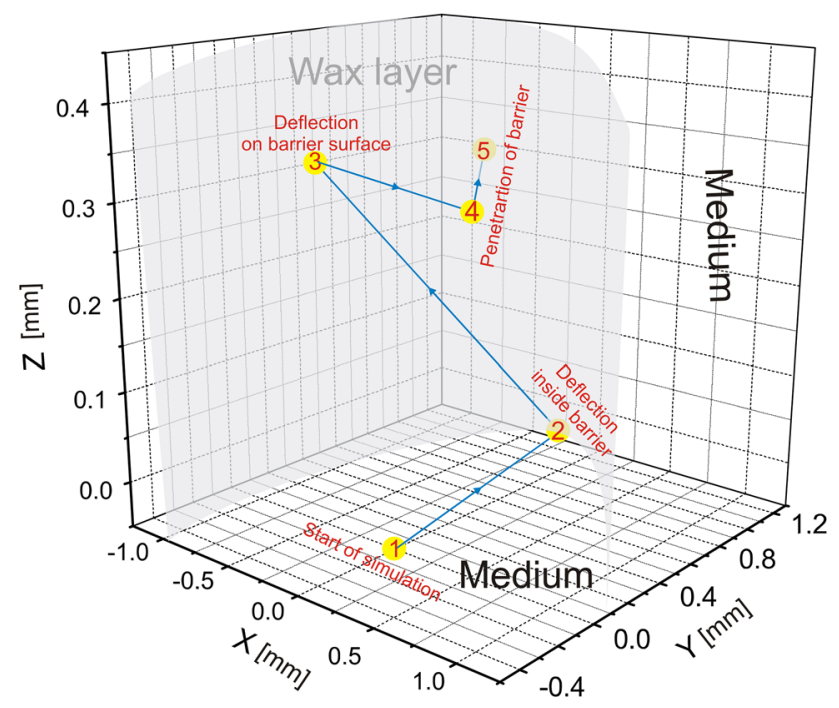

Fig. 6 The spatial structure determined for the $\mathrm{H}^{+}$ions passing through the cuticle barrier

smoothly (SI Table 2 and SI Figure 3, SI Figure 4 and SI Figure 5) and were negatively (and strongly) cross-correlated (insets in SI Figure 3, SI Figure 4 and SI Figure 5) (a negative correlation means that as one of the variables increased, the other tended to decrease and vice versa). All of these results are in agreement with the Monte Carlo predictions presented in Fig. 5.

Next, cross-correlations of elongation growth and $\mathrm{pH}$ for APW (IAA, FC) for all considered variants, which were calculated using Eq. (1), are presented in SI Figure 6, SI Figure 7 and SI Figure 8, Charts A-G, respectively. A second-order cross-correlation (I) that was calculated for the cross-correlation and time delay $(\mathrm{H})$ showed a maximum strength at zero minutes of abrasion.

In all cases (for APW, IAA, FC-SI Figure 6, SI Figure 7 and SI Figure 8, respectively) the second-order correlation (Chart I) for variant I was the strongest. Also, there were noticeable differences in the strength of this correlation for APW, IAA and FC cases. The weakest secondorder cross-correlation was observed for APW-1.17 units, the middle value of 2.78 units for IAA and the highest value of 3.25 units for FC. This is in agreement with the well-known fact that plant hormones affect growth, here reflected by the time delay- $\mathrm{pH} /$ growth correlation strength (SI Figure 7 and SI Figure 8, Chart I).

Eventually, the relative elongation, Eq. (2), which was calculated from the experimental data points using Fitexex software (Zajdel et al. 2016), is presented in SI Figure 9, SI Figure 10 and SI Figure 11 for all of the experimental conditions (APW, IAA, FC) and all of the considered variants (I-VII). The net (model) curve was split into linear $\left(\mathrm{H}^{+}\right.$diffusion) and nonlinear (cell wall synthesis) plots (see Pietruszka 2017 for interpretation). In addition, the incubation time, which originated from the linear extrapolation of A-G plots, is presented in Charts $\mathrm{H}$ of SI Figure 9, SI Figure 10 and SI Figure 11 (note the different behaviour for APW, IAA and FC). The $C$ and $D$ coefficients (the growth amplitude and diffusion rate, respectively) are presented as a function of the abrasion time in SI Figure 12, SI Figure 13 and SI Figure 14 and SI Table 3. The upper plots show growth amplitudes $C$ fitted by the Euler beta function (insets). A comparison with the work of Pietruszka and Haduch-Sendecka (2016a) leads to the preliminary conclusion that the cuticle layer thickness and temperature can apparently act interchangeably within the same scaled (normalised) range.

Auxiliary measurement results of $\mathrm{pH}$ as a function of time for the control (APW) and growth factor (IAA, FC) conditions parameterised by the experimental variants are presented in SI Figure 15, SI Figure 16 and SI Figure 17). A typical $\mathrm{pH}$ depletion-like behaviour in time was observed for all of the variants, though the system's response clearly differed in all of the cases.

The SEM images of abraded and non-abraded fragments of coleoptiles of $\times 50$ and $\times 1000$ magnification are presented in Figs. 10 and 11, respectively. The effect of Evans blue is presented in Fig. 12, while the effect of neutral red is shown in Fig. 13.

The data flow chart is presented in SI Diagram 1 in order to assist the reader. 

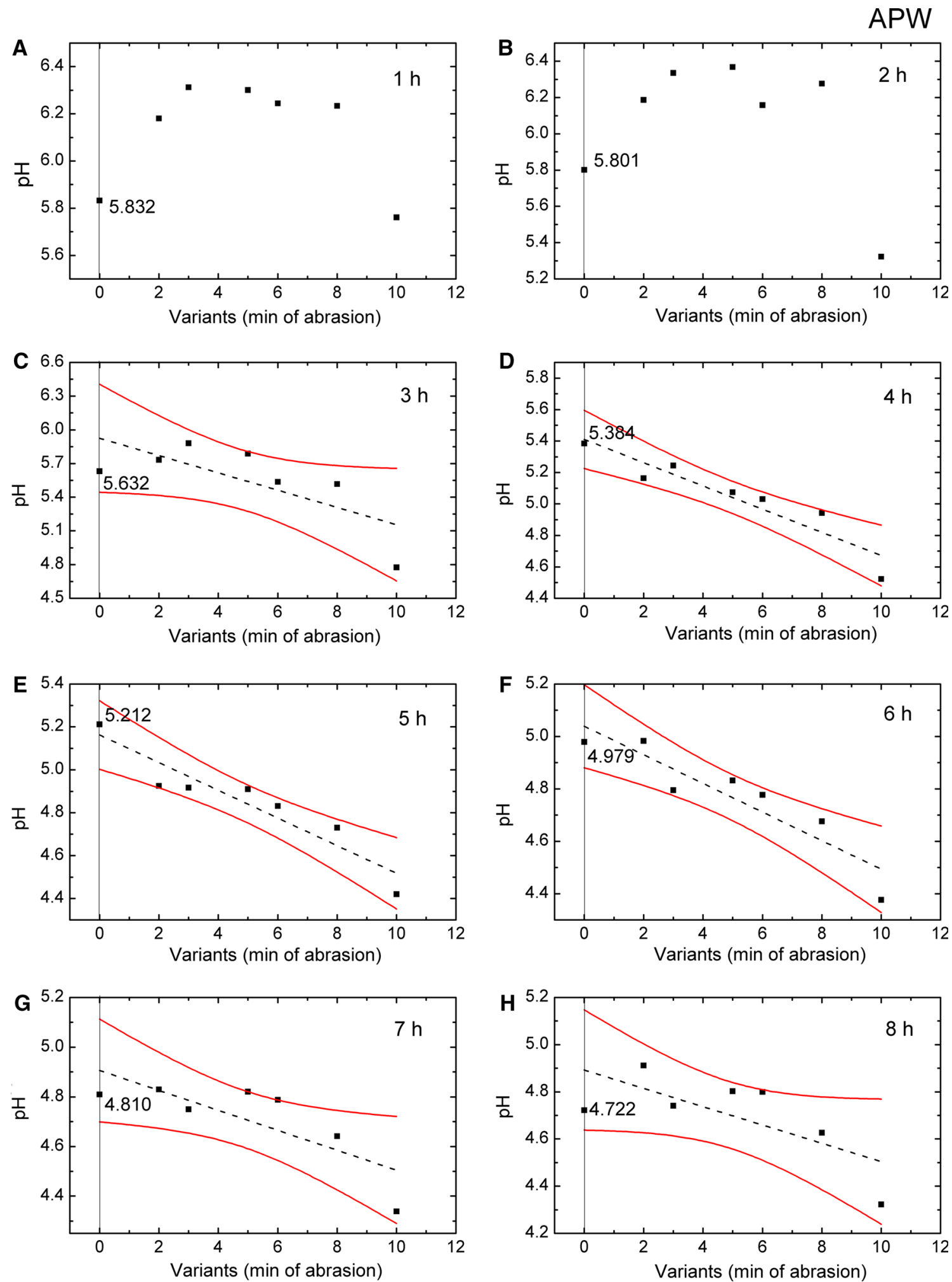

Fig. 7 Time cross-sections of $\mathrm{pH}$ as a function of the cuticle coverage of a coleoptile in control conditions (APW). The linear decrease in $\mathrm{pH}$ with the abrasion time (variants) corresponding to the numerical simulation presented in Fig. 6 is clearly visible for 3-7 h.

Confidence bands indicated at $95 \%$ of confidence level. For the continuously changing slope and intercept values, see SI Table 2 and SI Figure 3. The pH control value is specified in the charts 

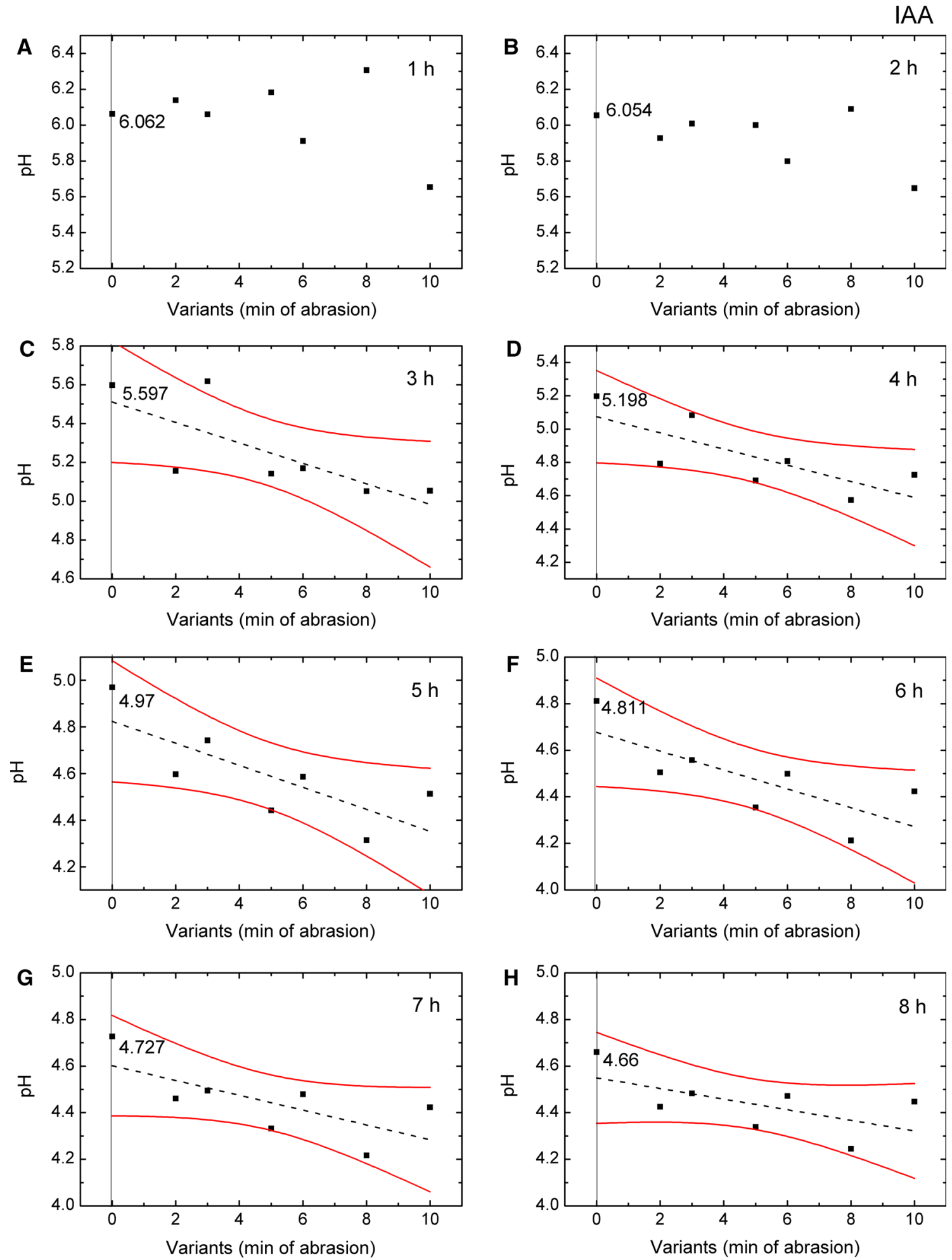

Fig. 8 Time cross-sections of $\mathrm{pH}$ as a function of the cuticle coverage of a coleoptile (IAA). The linear decrease in $\mathrm{pH}$ with the abrasion time (variants) corresponding to the numerical simulation presented in Fig. 6 is clearly visible for 3-7 h. Confidence bands

indicated at $95 \%$ of confidence level. For the continuously changing slope and intercept values, see SI Table 2 and SI Figure 4. The pH control value is specified in the charts 

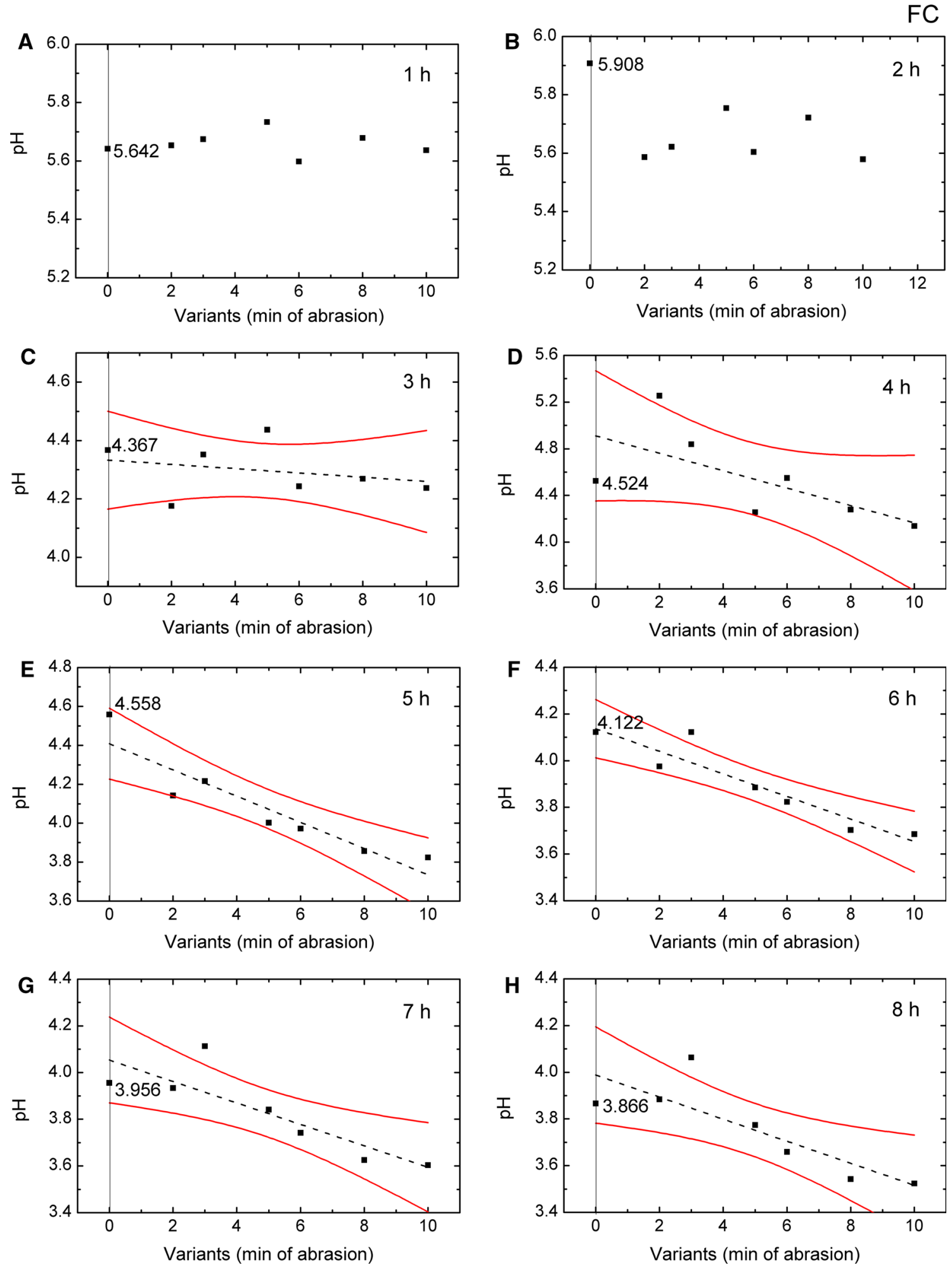

Fig. 9 Time cross-sections of $\mathrm{pH}$ as a function of the cuticle coverage of a coleoptile (FC). The linear decrease in $\mathrm{pH}$ with the abrasion time (variants) corresponding to the numerical simulation presented in Fig. 6 is clearly visible for $3-7 \mathrm{~h}$. Confidence bands

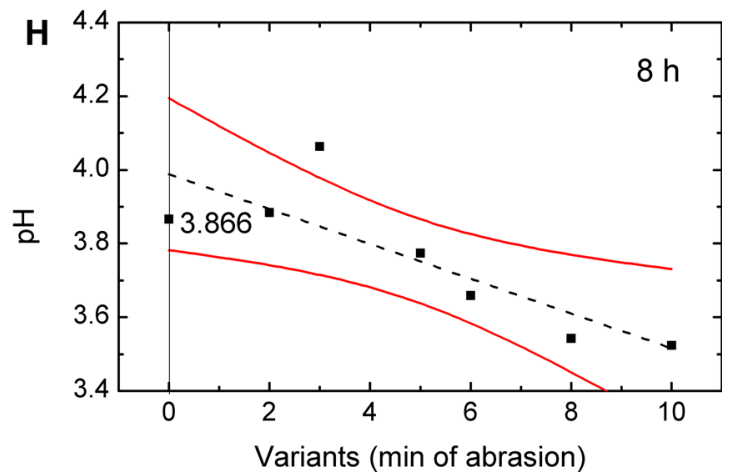

indicated at $95 \%$ of confidence level. For the continuously changing slope and intercept values, see SI Table 2 and SI Figure 5. The $\mathrm{pH}$ control value is specified in the charts 


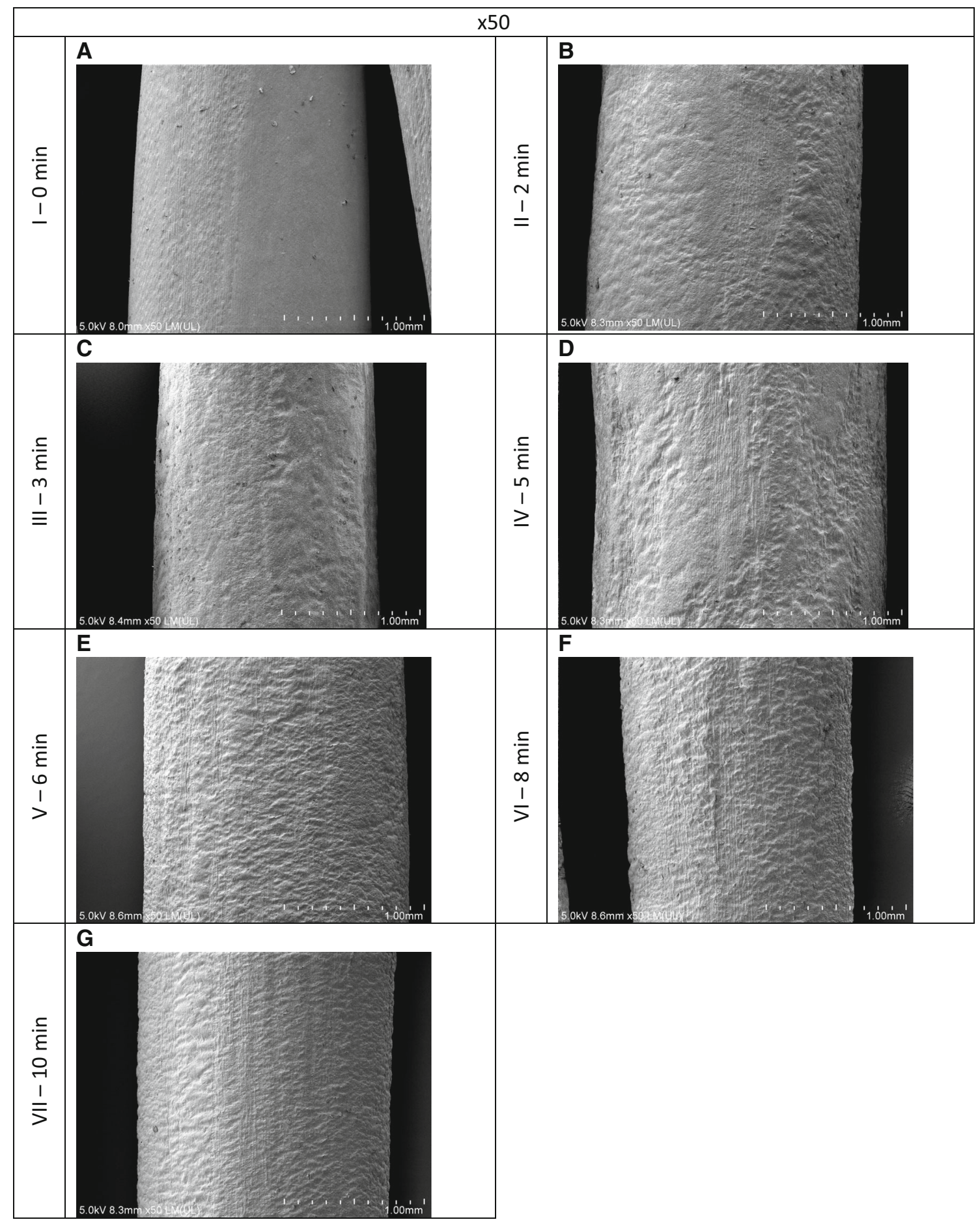

Fig. 10 Abraded (b-g) and non-abraded (a) cuticle coleoptile slides, magnification $\times 50$. Micrographs taken using a scanning electron microscope (SEM); representative fragment of a coleoptile has been

chosen. It was difficult to eliminate small artefacts appearing in the photos, which come inter alia from $\mathrm{SiC}$ and the remnants of exfoliation of the cuticle 


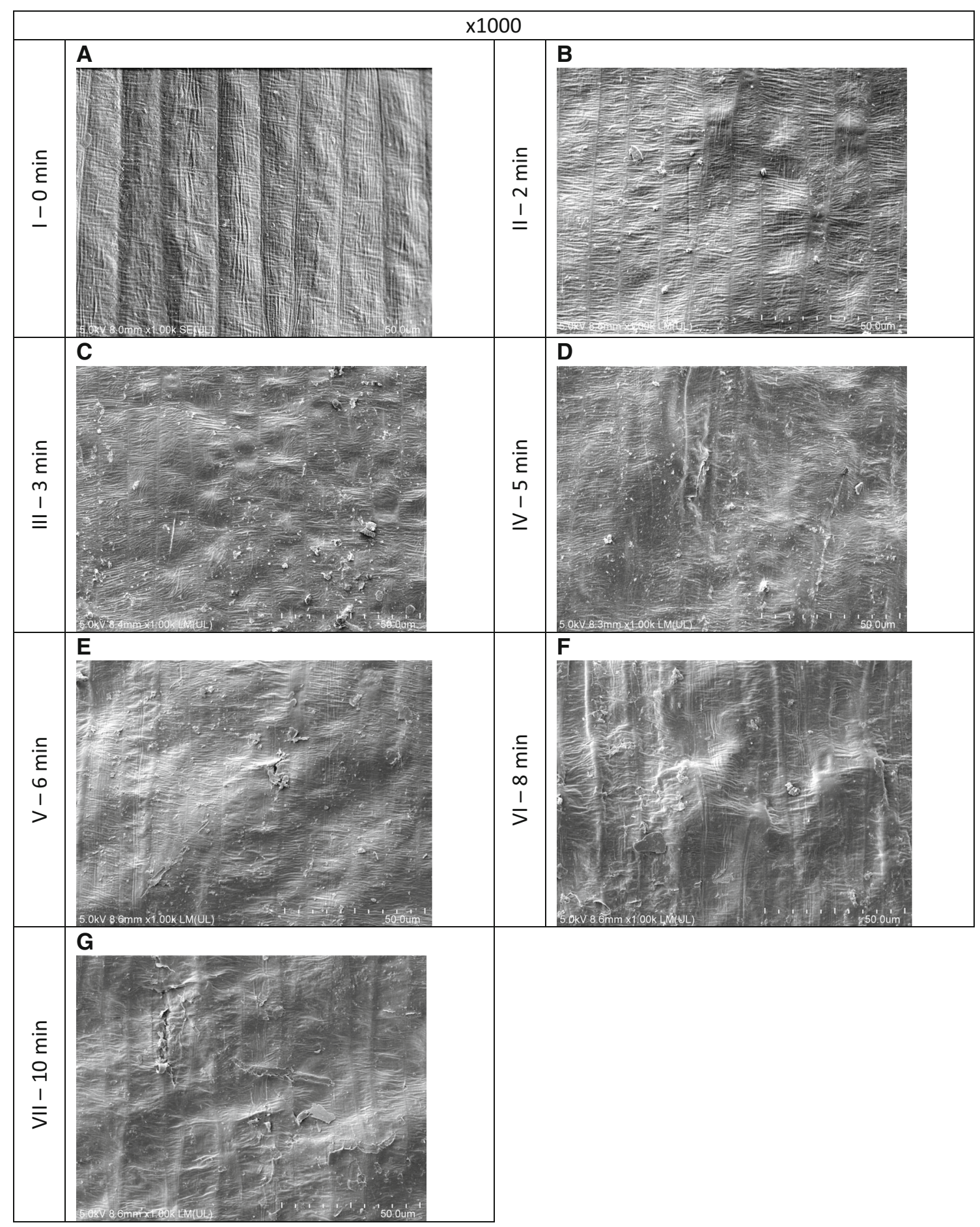

Fig. 11 Abraded (b-g) and non-abraded (a) cuticle coleoptile slides, magnification $\times 1000$; representative fragment of a coleoptile has been chosen. Note that along with the variant of abrasion, evenly and the pattern of cuticle becomes less and less visible with larger pleated. Micrographs taken using a scanning electron microscope distributed anticlinal ribs of epidermal cells are increasingly visible (SEM) 


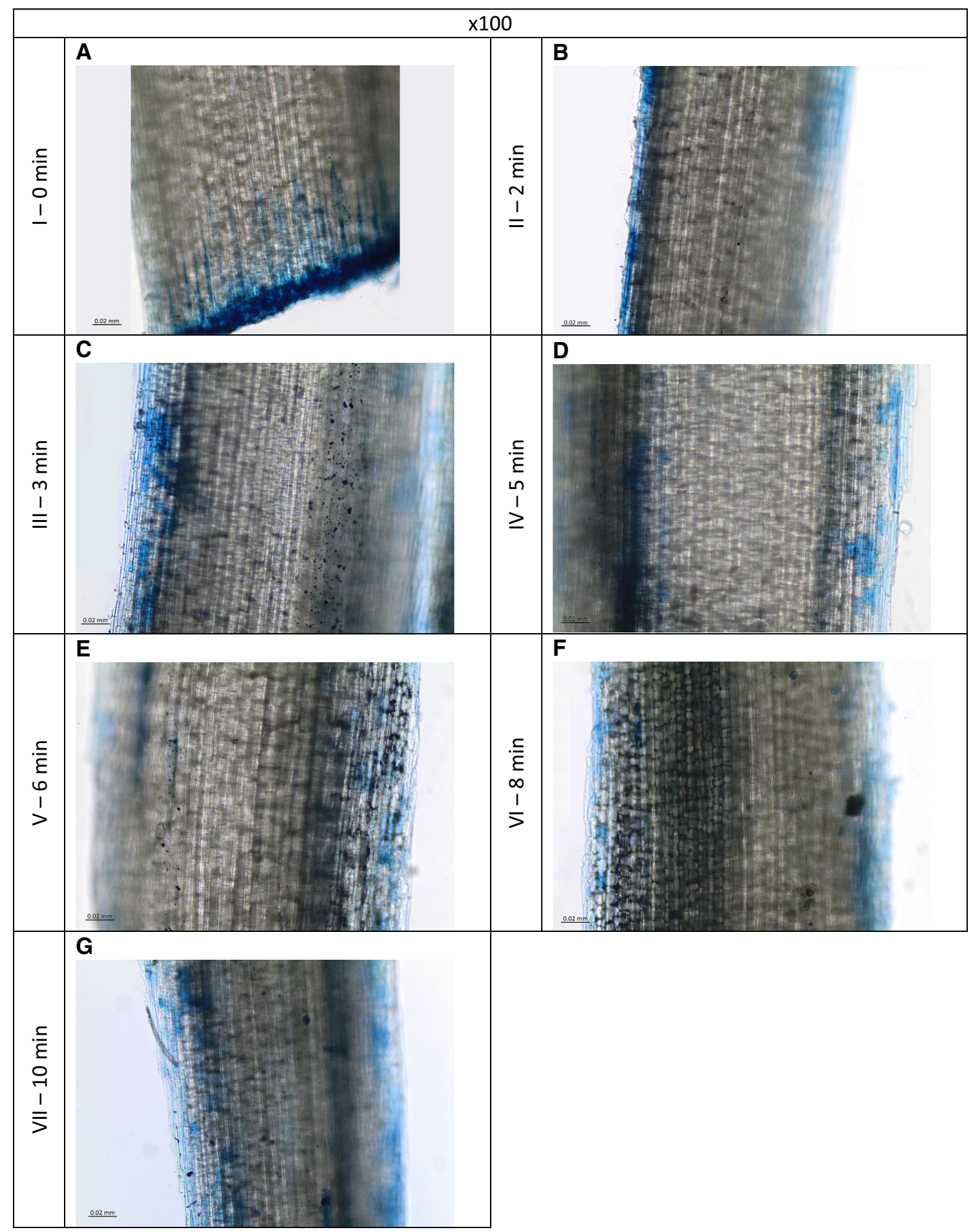

Fig. 12 Staining of the vertically oriented segments with Evans blue that enters dead cells only. Microscopic examination of the coleoptile surface showed that the abrasion method of cuticle produced only single epidermal cells that were damaged. The handmade crosssections at the cleavage site segment have a blue discoloration indicated dead cells 


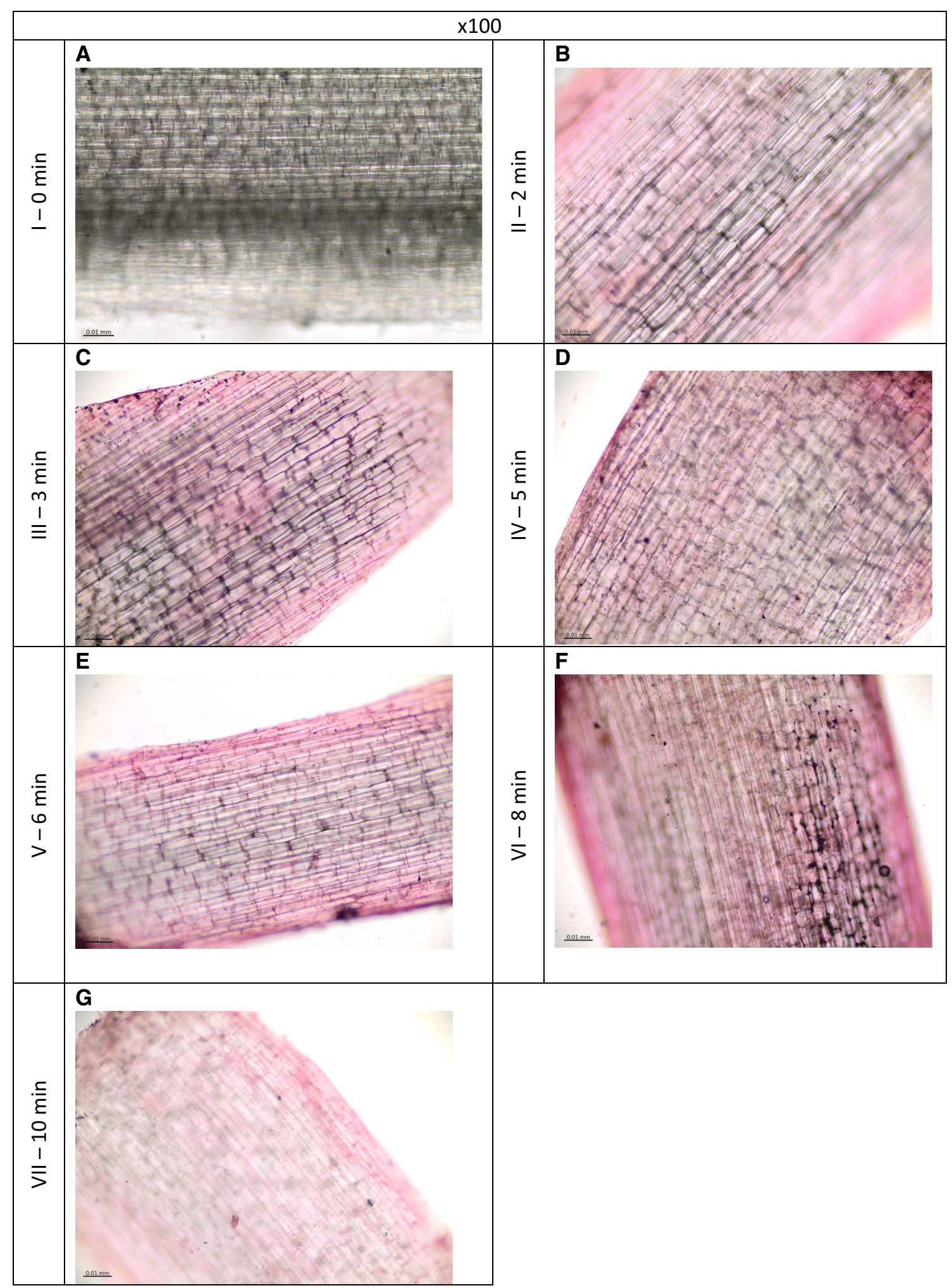

Fig. 13 Staining of the segments with neutral red that enters live cells only. Microscopic examination of the coleoptile surface showed how many cells are actually opened to the external solution 


\section{Discussion}

\section{Experiment}

This work deals with the effect of cuticle abrasion on growth and medium acidification for coleoptiles of maize. The correct way of abrasion has been an important issue in the discussion on the acid growth theory (Kutschera and Schopfer (1985) on one side, Cleland (1987, 1991), Hager (1991), Lüthen (1990), Peters et al. (1998) and others on the opposite side). Proper abrasion is needed to reduce the diffusion barrier imposed by cuticle and, therefore, an important experimental technique.

Research was carried out on abraded coleoptile segments because much of the literature reports on the examination of the cuticle barrier has been conducted using this method (Evans and Vesper 1980; Lüthen et al. 1990; Peters et al. 1992). In Figs. 7, 8 and 9, for all of the examined cases (APW, IAA, FC), the first $2 \mathrm{~h}$ of the experiment were the periods of acclimatisation after stress was induced in the tissue segments excised from the plant. These $2 \mathrm{~h}$ of preincubation time have also been observed by many researchers, e.g., in the article by Zajdel et al. (2016) where the growth was small or even negligible at this time point. In the remaining duration of the experiment, between 3 and $8 \mathrm{~h}$, the elongation of coleoptiles occurred along with the simultaneous acidification of the incubation medium. As an aside, we noted that a more intense cuticle abrasion (variants II-VII) caused a faster decrease in $\mathrm{pH}$ in the incubation medium. An analysis of $\mathrm{pH}$ between variants I-VII showed a linear decrease (Figs. 7, 8 and 9; 3-8 h). There was a clear discrepancy (difference) between the abraded coleoptiles and the intact ones. As was already shown by Lüthen et al. (1990), abraded coleoptiles are far more sensitive to the effect of external $\mathrm{pH}$ than undamaged ones. Therefore, as was observed in our research, the greater the degree of cuticle abrasion, the larger the decrease in $\mathrm{pH}$. Differences are also visible between the experiments with IAA and FC. In the paper by Lüthen et al. (1990), the decrease in $\mathrm{pH}$ in the presence of $\mathrm{FC}$ is more pronounced than that for IAA, which was also evident in our experiments (Figs. 8, 9). The differences in the decrease in $\mathrm{pH}$ for different variants is presented in SI Figure 3, SI Figure 4 and SI Figure 5, which presents the graphs for the intercept $a$ (upper plots), whereas the graphs that show the slope $b$ (angle of inclination) reveal the rate of $\mathrm{pH}$ decrease (lower plots), where this rate increased in time. Furthermore, a cross-correlation between intercept $a$ and slope $b$ shows a very high dependence between them. A similar cross-correlation between the growth rate and proton efflux rate was presented in the paper by Pietruszka and Haduch-Sendecka (2016a).

The cross-correlation for all of the variants (I-VII) in IAA and FC cases was much stronger (SI Figure 7, SI Figure 8) compared to all of the variants in the APW, which was almost halved (SI Figure 6). In Chart H in SI Figure 6, SI Figure 7 and SI Figure 8, the time delay is presented for all of the variants and cases of APW and IAA in which the cuticle abrasion time delay increases at the beginning and then begins to decrease. In the case of FC, it is almost at the same level with the exception of one variant of cuticle abrasion (variant VIII) for which a local decrease occurred. The second order cross-correlation for the time delay and the $\mathrm{pH} /$ growth cross-correlation strength in all of the cases formed a triangle (Chart I in SI Figure 6, SI Figure 7 and SI Figure 8). The weakest correlation occurred for APW, while it was much stronger for IAA and strongest for FC. This important result can be compared with the outcomes of Pietruszka and Haduch-Sendecka (2016a).

It is interesting that after the addition of growth factors (IAA or FC) in the second hour of the experiment, there was a steep decrease in $\mathrm{pH}$ (SI Figure 16 and SI Figure 17-a grey rectangle) in all of the variants of abrasion (I-VII). A similar decrease that occurs after the addition of IAA and FC is well known in the literature (Cleland 2010).

The multi-chart diagrams (SI Figure 9, SI Figure 10 and SI Figure 11) for APW, IAA and FC show a common share of nonlinear and linear part to reproduce the net (relative) elongation data using growth functional given by Eq. (1). The linear and nonlinear contributions can be interpreted in terms of $\mathrm{H}^{+}$diffusion and cell wall synthesis (Pietruszka 2017), respectively. The incubation time $\tau$ in different conditions is presented in Chart $\mathrm{H}$, which differs greatly in the considered cases. What also draws our attention is a noticeable minimum in the diffusion rate for APW (SI Figure 12), a local maximum for IAA case (SI Figure 13) and the linear dependence for FC (SI Figure 14), as a function of the abrasion time.

Besides, in the case of APW (Chart H in SI Figure 9), the incubation time $\tau$ extended along with the cuticle abrasion (for variant IV corresponding to $5 \mathrm{~min}$ ). This could be due to the additional stress caused by the abrasion procedure or even by a small amount of cuticle abrasion. Starting at variant $\mathrm{V}$, the incubation time $\tau$ was reduced, which could have been caused by a more intense cuticle abrasion, thus implying a greater flow of substances between the external environment and the tissue. This resulted in a faster response of growing plants and a shorter incubation time $\tau$, which was observed in the subsequent variants.

Interestingly, for the implementation of IAA, the incubation time $\tau$ was about $2 \mathrm{~h}$ (Chart $\mathrm{H}$ in SI Figure 10) for 
all of the variants of abrasion (time $\tau$ did not depend on the cuticle abrasion). Auxin is a plant hormone that naturally occurs in plant cells and, as it turned out, the plant response to auxin took place at approximately the same time $\tau$. Furthermore, it would appear that the presence of the outer epidermal cells is not essential for auxin effect as was confirmed by Peters et al. (1992), who stated that "Auxininduced $\mathrm{H}^{+}$-pump stimulation does not depend on the presence of epidermal cells in corn coleoptiles". Hence, it can be deduced that the absence/presence of a cuticle is even less important in this (IAA) case.

The action of FC showed yet another time $\tau$ dependence on the abrasion of the cuticle. The longer the cuticle abrasion time, the more reduced the incubation time $\tau$ (Chart $\mathrm{H}$ in SI Figure 11). This may be due to the fact that the removal of the cuticle could have caused easier access of FC to the cells and, consequently, a faster response of plant growth.

It is known from the literature that the mechanisms of action of IAA and FC differ significantly: IAA combines with $\mathrm{H}^{+}$-ATPase and activates it reversibly (cf. also SI Figure 13), while FC combines with $\mathrm{H}^{+}$-ATPase and activates it irreversibly (SI Figure 14). However, these complicated mechanisms are beyond the scope of this work and, therefore, we will avoid describing them in detail. In brief, it is accepted that protein ABP1 (Auxin Binding Protein 1) and TIR1/AFB (Transport Inhibitor Response 1/Auxin Fbox protein) are auxin's receptors (Lüthen 2015). The direct action of a receptor with the channel $\mathrm{H}^{+}$ATPase is assumed because $\mathrm{ABP}_{57}$ interacts directly with the proton pump and does not require a docking protein (Yong et al. 2001). In the case of protein TIR1, auxin is directly connected to the $\mathrm{SCF}^{\mathrm{TIR} 1}$. Protein TIR1 is part of the ubiquitin ligase (E3 enzyme) type SCF (SKP1-CullinF-box). On the other hand, the function of the fusicoccin receptor in plant cells is accomplished by the complex that is formed by the $\mathrm{C}$ terminus of the $\mathrm{H}^{+}$-ATPase and the dimer of 14-3-3 protein (Olivari et al. 1998). Fusicoccin attachment to the complex causes an irreversible $\mathrm{H}^{+}$ATPase activation because FC, jointly with the 14-3-3 protein, effectively blocks the access of phosphatases to phosphorylated threonine (Olsson et al. 1998). Fusicoccin is a highly effective activator of plasma membrane $\mathrm{H}^{+}$ATPase. During phytotoxic-induced growth, a strong acidification of the incubation environment occurs due to the protons being pumped out of the cell (Higgins et al. 2007).

Although the mechanisms of action of IAA and FC differ, the final effect is similar (proton extrusion). In our research, we observed a cross-correlation between the time delay and the strength of the $\mathrm{pH} /$ growth cross-correlation (SI Figure 7 and SI Figure 8-Chart I), which was in the shape of a (autocorrelation) triangle for both cases
(Pietruszka and Haduch-Sendecka 2016a). Interestingly, different results were also obtained for the coefficients $C$ and $D$, which are presented in SI Figure 13 and SI Figure 14, which show a maximum of diffusion rate at 2 min abrasion for IAA and the linear increase of diffusion for FC.

In the above context, it is also appealing to compare the amplitude $C$ and diffusion rate $D$ that are presented in Pietruszka and Haduch-Sendecka (2016a), where both coefficients are plotted as a function of temperature. Indeed, a direct comparison suggests that the cuticle (thickness) constitutes a potential barrier for $\mathrm{H}^{+}$ions (SI Figure 12, SI Figure 13 and SI Figure 14) that is similar to the action of temperature at physiological range. Penetration of the cuticle barrier by protons increases with temperature or abrasion time, as it can be intuitively expected.

Diffusion resistance of cuticle is different for dissimilar species. It depends on the structure, density and number of cutin layers and waxes present in the outer walls of the epidermis, and also on the thickness of the cuticle. Leaves of plants adapted to live in dry air and dry soil tend to have thicker layers of cuticle and waxes than leaves of plants growing in high humidity conditions. Therefore, an important issue in the discussion on the acid growth theory has been the correct way of cuticle abrasion. In the discussion in the 1990s there were two opposed camps, and there were also several methods for cuticle abrasion of the coleoptiles. On the one side, Kutschera and Schopfer (1985) abraded coleoptiles with wet emery cloth or peeled the samples. On the opposite side, Cleland (1987, 1991) abraded the sample with emery powder or peeled with fine jewellers forceps, respectively, Hager (1991) abraded coleoptiles between thumb and forefinger with the $\mathrm{SiC}$ paste, Lüthen (1990) abraded the samples with SiC powder, and Peters et al. (1998) pulled coleoptiles through loops of fine emery cloth. As it was stated before, proper abrasion is needed to reduce the diffusion barrier imposed by the cuticle and therefore it is an important experimental technique. For this reason, bearing in mind the existing methods of abrasion of the cuticle, a new method has been developed in the article. In our experiment, it turned out that the method produced only single cells that were damaged (Figs. 12, 13).

\section{Theory}

In this work, the GEANT4 Monte Carlo simulation toolkit with the GEANT4-DNA module that is dedicated to modelling the interactions that are induced by ionising radiation on the DNA damage was used. The implemented models of the physical processes make it possible to perform simulation in the liquid water and other biological materials. Calculations of the ion transport can be 
simulated in thin layers within the applied models. The literature data indicate that the GEANT4-DNA module has been used for microdosimetry simulations (Chauvie et al. 2007), for simulations of Auger electron emission from nanometer-sized gold targets (Incerti et al. 2016), in computational radiation biophysics (Kuncic 2015), for proton transport in water and DNA components (Champion et al. 2013) and several others. The application of this software to the calculation of the transport of $\mathrm{H}^{+}$ions through the cuticle layer, which is treated as a potential barrier, is quite innovative. Moreover, it is a new approach that was used for the considered problem.

The software that was used offers the possibility to do simulations of hydrogen interactions with the nuclei as well as with the electrons of single atoms of a biological medium in an energy range with a relatively low energy threshold below which the incident ions are stopped and the kinetic energy is deposited locally. The minimum initial energy of $\mathrm{H}^{+}$ions is equal to $100 \mathrm{eV}$. However, the hydrogen ion energy after interactions can be much less. This energy depends on the physical process that is used. In the case of the atom ionisation, the simulation continues until the $\mathrm{H}^{+}$ions are stopped when the G4DNARuddIonisationModel class or its extended version is applied. Electronic excitations using the G4DNAMillerGreenExcitationModel class have an energy threshold of $10 \mathrm{eV}$, whereas nuclear elastic scattering can be simulated to $100 \mathrm{eV}$.

In the presented work the movement of $\mathrm{H}^{+}$ions through the cuticle layer was simulated. In the simulations the directions and energy of the $\mathrm{H}^{+}$ions reaching the cuticle were estimated according to the Monte Carlo method. This way the transport of the $\mathrm{H}^{+}$ions through the cuticle layer was realized for the case without IAA and FC. In the simulation the diffusion mechanism was reconstructed by a set of the primary energy and the direction of a movement of $\mathrm{H}^{+}$ions according to the Monte Carlo method. However, the movement of a $\mathrm{H}^{+}$-ion across the apoplast strongly differs from simple diffusion, as $\mathrm{H}^{+}$-ions are charged and they interact with charges in the cell wall, which is a large ion exchanger. The number of charges will depend on the $\mathrm{pH}$ in the apoplast (Grignon and Sentenac 1981, 1991) and thus will differ in the cases of APW, IAA and FC. Obviously, the model is only a first approximation of the movement of protons across the apoplast.

The used software does not permit simulations of $\mathrm{H}^{+}$ ion interactions with whole molecules of a medium. Perhaps, the next versions of the GEANT4 code will provide such possibilities as well as permit the simulations to be started from a lower energy of $\mathrm{H}^{+}$ions. In spite of these facts, the obtained results indicate that there is quite a good agreement between the simulations and the data from the experiment that was performed. The linear decrease in $\mathrm{pH}$ with the abrasion time (variants) that is shown in Figs. 7, 8 and 9 corresponds to the dependence that was determined based on the numerical Monte Carlo simulations that are presented in Fig. 5. What is more, the spectra of $\mathrm{H}^{+}$ions passing through the cuticle barrier, which are presented in Fig. 4, have two clear peaks. The interpretation of this fact can be that these peaks may be related to the two maxima that are visible in the dependence of the growth amplitude coefficient $(C)$ and diffusion rate $D$, versus the abrasion time that is presented in SI Figure 12, SI Figure 13 and SI Figure 14.

Thus, the software that was used makes it possible to obtain reliable results. Therefore, it is worth monitoring the progress of the GEANT4 simulation toolkit.

\section{Conclusions}

In the past 400 years, physics has achieved great success in both theory and experimentation for determining the fundamental structure of matter and energy. The next great step in the evolution of science will be exploring theoretical constructs in order to yield specific predictions in biology, in particular, plant physiology. Here, we propose that questions about plant physiology can be approached from a method that is well established in physics, the Monte Carlo simulation. This method would allow us to develop a quantitative approach to $\mathrm{H}^{+}$ions penetrating micrometer-thin cuticle layer and to compare it with experimental results. By following the chain of argument, we also considered the possibility that (based on our own experiment) the estimated diffusion rates of $\mathrm{H}^{+}$ions can be related to the Monte Carlo predictions concerning $\mathrm{H}^{+}$ions spectra. We also formulated the possible relationship between the environmental temperature and the thickness of the cuticle layer in the context of the kinetics of $\mathrm{H}^{+}$ions. This can potentially be of interest in the perspective of a study by Jung et al. (2016), where researchers describe a major thermosensory role of phytochromes (red light receptors) during the night.

What we propose in this paper is a natural and straightforward extension of the usual growth experiments to quantitative measurements of different experimental variants and growth conditions (APW, IAA and FC). However, a framework that integrated data from multiple disciplines could help us develop a broader context to plant cell extension growth than what is possible from a single field alone.

Author contribution statement $M P$ conceived the original screening and research plans, $A K$ performed Monte Carlo simulations, $M O$ performed all of the experiments and samples preparations for SEM, $M P$ and $M O$ designed 
the experiments and analysed the data, $M P$ supervised the experiments, $M P$ conceived the project and wrote the article with contributions of all the authors, $M P$ supervised and complemented the writing. All authors reviewed the manuscript.

Acknowledgements We thank dr Jagna Karcz, Scanning Electron Lab., Faculty of Biology and Environment Protection, University of Silesia, for SEM images.

Open Access This article is distributed under the terms of the Creative Commons Attribution 4.0 International License (http://crea tivecommons.org/licenses/by/4.0/), which permits unrestricted use, distribution, and reproduction in any medium, provided you give appropriate credit to the original author(s) and the source, provide a link to the Creative Commons license, and indicate if changes were made.

\section{References}

Bidhendi AJ, Geitmann A (2015) Relating the mechanics of the primary plant cell wall to morphogenesis. J Exp Bot 67:449-461

Braidwood L, Breuer C, Sugimoto K (2014) My body is a cage: mechanisms and modulation of plant cell growth. New Phytol 201:388-402

Champion C, Incerti S, Tran HN, Karamitros M, Shin JI, Lee SB, Lekadir H, Bernal M, Francis Z, Ivanchenko V, Fojón OA, Hanssen J, Rivarola RD (2013) Proton transport in water and DNA components: a Geant4 Monte Carlo simulation. Nucl Instrum Meth B 306:165-168

Chauvie S, Francis Z, Guatelli S, Incerti S, Mascialino B, Moretto P, Nieminen P, Pia MG (2007) Geant4 physics processes for microdosimetry simulation: design foundation and implementation of the first set of models. IEEE Trans Nucl Sci 54:2619-2628

Cleland RE (1991) The outer epidermis of Avena and maize coleoptiles is not a unique target for auxin in elongation growth. Planta 186:75-80

Cleland RE (2010) Auxin and cell elongation. Plant hormones. Springer, Netherlands, pp 204-220

Cleland RE, Cosgrove D, Tepfer M (1987) Long-term acid-induced wall extension in an in vitro system. Planta 170:379-385

Cosgrove DJ (2001) Wall structure and wall loosening. A look backwards and forwards. Plant Phys 125:131-134

Cosgrove DJ (2005) Growth of the plant cell wall. Nature 6:850-860

Evans ML, Vesper MJ (1980) An improved method for detecting auxin-induced hydrogen ion efflux from corn coleoptile segments. Plant Physiol 66:561-565

Grignon C, Sentenac H (1991) $\mathrm{pH}$ and ionic conditions in the apoplast. Ann Rev Plant Physiol Plant Mol Biol 42:103-128

Hager A (2003) Role of the plasma membrane $\mathrm{H}^{+}$-ATPase in auxininduced elongation growth: historical and new aspects. J Plant Res 116:483-505

Hager A, Debus G, Edel H-G, Stransky H, Serrano R (1991) Auxin induces exocytosis and the rapid synthesis of a high-turnover pool of plasma-membrane $\mathrm{H}^{+}$-ATPase. Planta 185:527-537

Higgins R, Lockwood T, Holley S, Yalamanchili R, Stratmann J (2007) Changes in extracellular $\mathrm{pH}$ are neither required nor sufficient for activation of mitogen-activated protein kinases (MAPKs) in response to system in and fusicoccin in tomato. Planta 225:1535-1546
Incerti S, Suerfu B, Xu J, Ivantchenko V, Mantero A, Brown JMC, Bernal MA, Francis Z, Karamitros M, Tran HN (2016) Simulation of Auger electron emission from nanometer-size gold targets using the Geant4 Monte Carlo simulation toolkit. Nucl Instrum Meth B 372:91-101

Jung JH, Domijan M, Klose C, Biswas S, Ezer D, Gao M, Khattak AK, Box MS, Charoensawan V, Cortijo S, Kumar M, Grant A, Locke JC, Schäfer E, Jaeger KE, Wigge PA (2016) Phytochromes function as thermosensors in Arabidopsis. Science 354:886-889

Kuncic Z (2015) Advances in computational radiation biophysics for cancer therapy: simulating nano-scale damage by low-energy electrons. Biophys Rev Lett 10:25-36

Kutschera U (1994) The current status of the acid-growth hypothesis. New Phytol 126:549

Kutschera U (2003) Auxin-induced cell elongation in grass coleoptiles: a phytohormone in action. Curr Topics Plant Biol 4:27-46

Kutschera U, Schopfer P (1985) Evidence for the acid-growth theory of fusicoccin action. Planta 163:494-499

Lüthen H (2015) What can we learn from old auxinology. J Plant Growth Regul 34:702-707

Lüthen H, Bigdon M, Böttger M (1990) Reexamination of the acid growth theory of auxin action. Plant Physiol 93:931-939

Olivari C, Meanti C, De Michelis MI, Rasi-Caldogno F (1998) Fusicoccin binding to its plasma membrane receptor and the activation of the plasma membrane $\mathrm{H}^{+}$-ATPase. Plant Physiol 116:529-537

Olsson A, Svennelid F, Ek B, Sommarin M, Larsson C (1998) A phosphothreonine residue at the $\mathrm{C}$-terminal end of the plasma membrane $\mathrm{H}^{+}$-ATPase is protected by fusicoccin-induced 14-33 binding. Plant Physiol 118:551-555

Peters WS, Richter U, Felle HH (1992) Auxin-induced $\mathrm{H}^{+}$-pump stimulation does not depend on the presence of epidermal cells in corn coleoptiles. Planta 186:313-316

Peters WS, Lüthen H, Böttger M, Felle H (1998) The temporal correlation of changes in apoplast $\mathrm{pH}$ and growth rate in maize coleoptile segments. Aust J Plant Physiol 25:21-25

Pietruszka M (2012) A biosynthesis/inactivation model for enzymatic WLFs or non-enzymatically mediated cell evolution. J Theor Biol 315:119-127

Pietruszka M (2017) $\mathrm{pH} / \mathrm{T}$ duality - wall properties and time evolution of plant cells. Front Plant Sci, under review. See arXiv:1505.00327 for the 'equation of state'. Also: Correlations and Coherence at Different Scales, 40th Inter Conf Theor Phys, 4-9 Sept 2016, Ustroń, Poland, Abstracts, p. 129

Pietruszka M, Haduch-Sendecka A (2016a) Effective diffusion rates and cross-correlation analysis of "acid growth" data. Acta Physiol Plant 38(53):1-17

Pietruszka M, Haduch-Sendecka A (2016b) A quantitative report on the impact of chloride on the kinetic coefficients of auxininduced growth: a numerical contribution to the "acid growth hypothesis". SpringerPlus 5(1978):1-25

Pietruszka M, Lewicka S (2007) Anisotropic plant growth due to phototropism. J Math Biol 54:45-55

Sentenac H, Grignon C (1981) A model for predicting ionic equilibrium concentrations in cell-walls. Plant Physiol 68:415-419

Yong SK, Jung KM, Donghern K, Jin J (2001) A soluble auxinbinding protein, $\mathrm{ABP}_{57}$. J Biol Chem 6:10730-10736

Zajdel P, Pietruszka M, Haduch-Sendecka (2016) Application of the effective formula of growth functional to quantitative description of growth of plant cells. Acta Physiol Plant 38:216, 1-9. https:// github.com/pawelzajdel/fitexex 